\title{
101 ADVANCE CARE PLANNING IN PAEDIATRICS: THE CARE PROVIDERS' PERSPECTIVE
}

J D Lotz, ${ }^{1}$ R J Jox, ${ }^{2}$ G D Borasio, ${ }^{3}$ M Führer ${ }^{1}{ }^{1}$ Coordination Center for Pediatric Palliative Care, University Children's Hospital Munich, Germany; ${ }^{2}$ Institute of Ethics, History and Theory of Medicine, Ludwig-Maximilians University Munich, Germany; ${ }^{3}$ Service des Soins Palliatifs, Centre Hospitalier Universitaire Vaudois, Lausanne, Switzerland

\subsection{6/bmjspcare-2013-000491.101}

Background Research on advance care planning (ACP) has focused on adults, but ACP is also being practiced in pediatrics (pACP). Health care providers may have to deal with unknown diagnoses and prognoses, patients without decision making capacity and a complex social network. However, research on pACP has widely neglected the professionals' perspective.

Aim Investigating the experiences and needs of both the facilitators and the recipients of ACP.

Methods We conducted 17 semi-structured interviews with experts in the care of severely ill children and adolescents. Interviewees were from different professions, settings and institutions. The interviews were evaluated using inductive categorisation and thematic comparative analysis.

Results Pediatric ACP concerns all care providers. Particularly important issues are: An interdisciplinary care conference, repeated discussions with the parents, a continuous point of contact as well as emergency planning. Advance directives (AD) should be individualised, validated by the treating physician (shared decision making) and be available. Perceived challenges are: Parents' reluctance to think ahead, uncertain prognoses and uncertainty about involving the child. Conflicting interests exist between emergency physicians who are uncomfortable with accepting a child's $\mathrm{AD}$ when they are called in, and non-medical providers who are uncomfortable with accepting an AD without the verification by a physician.

Discussion This is the first European study that investigates the perspective of professionals on pACP. There is a need for pACP, but the perspectives differ between care providers.

Conclusion This study helps in understanding the needs of the different care providers as well as barriers to $\mathrm{pACP}$. 